\title{
Strain energy storage and dissipation rate in active cell mechanics
}

\author{
A. Agosti, ${ }^{1}$ D. Ambrosi, ${ }^{2}$ and S. Turzi ${ }^{1}$ \\ ${ }^{1}$ Dipartimento di Matematica, Politecnico di Milano, Piazza Leonardo da Vinci 32, 20133 Milano, Italy \\ ${ }^{2}$ DISMA, Politecnico di Torino, corso Duca degli Abruzzi 24, 10129 Torino, Italy
}

(Received 22 December 2017; published 22 May 2018)

\begin{abstract}
When living cells are observed at rest on a flat substrate, they can typically exhibit a rounded (symmetric) or an elongated (polarized) shape. Although the cells are apparently at rest, the active stress generated by the molecular motors continuously stretches and drifts the actin network, the cytoskeleton of the cell. In this paper we theoretically compare the energy stored and dissipated in this active system in two geometric configurations of interest: symmetric and polarized. We find that the stored energy is larger for a radially symmetric cell at low activation regime, while the polar configuration has larger strain energy when the active stress is beyond a critical threshold. Conversely, the dissipation of energy in a symmetric cell is always larger than that of a nonsymmetric one. By a combination of symmetry arguments and competition between surface and bulk stress, we argue that radial symmetry is an energetically expensive metastable state that provides access to an infinite number of lower-energy states, the polarized configurations.
\end{abstract}

DOI: 10.1103/PhysRevE.97.052410

\section{INTRODUCTION}

Living cells at rest observed in vitro on a flat substrate typically exhibit a rounded or an elongated shape. A transition from the symmetric to the polar configuration can be triggered by several factors [1], including an externally enforced strain [2] and a modulated myosin activity [3,4]. The polarization process can sometimes be instrumental toward initiation of migration, but a polarized state at rest is likely observed too.

In a symmetric cell the cytoskeleton has no apparent symmetry at a microstructural level: there is no array-type organization at a sarcomere level like in striated muscles. However, several possible mechanisms of transmission of the forces [1] determine a tensional stress that is essentially of radial direction in bulk, supplemented by a hoop stress component near the membrane, produced by the cortex [5]. Radially symmetric cells are said to be nonpolarized; conversely, the shape of a polarized cell has a symmetry axis, the actin fibers and the stress in the cytoskeleton are largely aligned along the axial direction.

Even when a cell is apparently at rest, a continuous process of material reorganization occurs inside. The active stress generated by the myosin motors stretches and displaces the polymeric network and creates a tensional pattern largely dictated by the fibers' alignment. From an energetic point of view, the active stress generates both strain energy storage and dissipative mass flow [6].

The surface and volume stress sources in a cell reflect the organization of the polymeric network in two architectures: actomyosin cortex along the membrane and the molecular motors attached to the cytoskeleton. Surface and bulk stress compete with the dendritic growth near the membrane, which can generate destabilization of the symmetric configuration. Tuning the amount of recruited tensile units [4], when the active stress dominates the cell is symmetric, while polarization (and migration) emerges for weaker activity of myosin.
An investigation of the mechanical balances involved in the two geometric configurations introduced above is intimately related to a precise statement of the rheological properties of the cytoskeleton. The rheological properties of the myosin cross-linked actin cytoskeleton, the basic structural element of a living cell, are complex. In the simplest approximation it can be represented as a viscoelastic Kelvin-type material, exhibiting solid-type behavior at short times and $(\sim 10 \mathrm{~s})$ and flowing like a viscous fluid on a longer time scale [7]. The actual rheology is, however, more complex: cyclic loads of in vitro cross-linked actin show a very nonlinear elastic behavior that undergoes softening in time because of a plastic reorganization $[8,9]$ likely due to a dynamic evolution of the mutual bonds between polymers. An analogous transition from elastic to viscoplastic regime is observed in vivo for optically stretched cells [10].

The deep insight reached in the understanding of the complex rheology of cross-linked actin networks is far from being translated in the mechanics of living cells; however, a representation of the cell as a purely mechanical system has been proven to be sufficient to reproduce a number of observed behaviors. Most common models retain only the linear and nonlinear fluidlike properties of the active material [7,11-13]; this framework has been particularly successful in producing nonsymmetric patterns of velocity field that account for the motion of cell-like droplets. Nonlinear elastic properties of the cytoskeleton are often relegated to the linear regime, where they account for transient behavior, the focus being on the flow at the steady state. Mean-stress arguments provide an inequality that relates the tension generated by the actomyosin ring and the force per unit surface in the body of the cell [14]. Kozlov and Mogilner [15] study the polarization and bistability of a cell fragment on the basis of surface vs volumetric energy contributions at variance of the geometry of the domain. They sketch the cell as a circle cut by an arc of variable length that represents the rear of the lamellipodium in the polarized 
configuration. While the free energy of the leading edge in the cytoskeleton and in the rear bundle grow linearly vs radius, area, and length, respectively, their linear combination is a complex function of the degree of polarization of the cell. This interplay between energetic and geometrical arguments can explain the existence of two equilibrium configurations.

In this paper we address a comparison of symmetric vs polarized cells from a purely mechanical point of view, in a solid (energy-preserving) and fluid (fully dissipative) setting. We disregard the inner mechanisms that drive the transition from one geometric configuration to the other (see Ref. [1] for a discussion) and we focus on the energetic balance that characterizes each of them. Strain energy storage is calculated on the basis of a hyperelastic model, while energy dissipation is based on a purely viscous one. This full decoupling between the physical regimes corresponds to consider the mechanical system at a long time scale, when both the stored elastic energy and the dissipation rate are constant in time. The quantitative evaluation obtained by numerical integration of the balance equations provides physical arguments for an insight of the energetic peculiarities of the geometrical configurations.

\section{STRAIN ENERGY STORAGE: THE ELASTIC MODEL}

In this work we adopt a very simple nonlinear strain energy density of compressible neo-Hookean material

$$
W(\mathrm{~F})=\frac{E}{2}[\mathrm{~F} \cdot \mathrm{F}-2 \log (\operatorname{det} \mathrm{F})-2],
$$

where $E$ is an elastic modulus and $\mathrm{F}$ is the tensor gradient of deformation. The strain energy (1) corresponds, in a small strain regime, to an isotropic linear material with shear modulus $E$ and infinite compressibility (zero bulk modulus). Volume variations are only penalized logarithmically at large strains.

First variation of the strain energy density yields the Piola stress. The balance of forces is to be complemented, for an active material, by the active component of the Piola stress tensor yielding:

$$
\mathrm{P}(\mathrm{F})=E\left(\mathrm{~F}-\mathrm{F}^{-T}\right)+\alpha \frac{\operatorname{det}(\mathrm{F})}{\sqrt{\mathrm{F} \mathbf{a}_{o} \cdot \mathrm{F} \mathbf{a}_{o}}} \mathrm{~F} \mathbf{a}_{o} \otimes \mathbf{a}_{o},
$$

where $\mathbf{a}_{o}$ is the reference direction of aligned actin fibers or, more in general, the direction of the active stress as emerging by a collective dynamics. The coefficient $\alpha$ modulates the amplitude of the active stress. From (2) it results a symmetric Cauchy active stress tensor, which is a linear function of the stretch, oriented according to the direction of the actual direction of the unit vector $\mathbf{a}_{o}$; pull back of the Cauchy stress to the material coordinates yields the Piola stress in Eq. (2) [16].

In a similar way, the actin cortex tension generates a compression at the boundary that acts as a constant pressure $\kappa$; in material coordinates the Nanson formula gives the boundary condition

$$
\mathrm{P}(\mathrm{F}) \mathbf{n}_{0}=-\kappa(\operatorname{det} \mathrm{F}) \mathrm{F}^{-T} \mathbf{n}_{0},
$$

where $\mathbf{n}_{0}$ is the outgoing normal to the boundary in the reference configuration.

We remark that the generic functional dependence of the active stress in a living cell on the deformation of the material itself is well known when a sarcomeric organization is present: aligned fibers generate a stress that grows linearly with the stretch when it is moderate, up to a plateau. Analogous relationships are not yet established when there is no alignment, like in the fibers of the cytoskeleton. An analysis of the microscopic dynamics of polar actin filaments activated by molecular motors points out the role of fibers' deformation in the emerging behavior for disordered networks too [17]; the macroscopic stress is therefore expected to depend on the deformation of the microstructure. Notwithstanding the lack of an ordered structure in actin filaments, the myosin contractility can produce very regular patterns in displacement and stress [18]; the mechanism that orchestrates such a regular dynamics starting from a random orientation and its interplay with the cell membrane are a very active area of research [1]. Here we will adopt orientation of the stress in precise direction not because we are considering array-type organization of the microstructure, but because we assume an ordered tensional pattern generated from a small-scale dynamics, which is immaterial for our purposes.

We sketch the shape of a polarized cell as a square of (reference) length $L_{o}$ with all fibers aligned in the axial direction, while a symmetric cell is represented as a circle of (reference) radius $R_{0}$, all the fibers being aligned in radial direction. We assume that a cell has the same volume in the two geometrical configurations:

$$
\pi R_{0}^{2}=L_{o}^{2}
$$

\section{A. Polarized cell}

Consider an unloaded squaredlike cell made of hyperelastic material, subject to bulk fiber-aligned active stress and active cortex stress at the boundary, proportional to the strain in the tangential direction. The resulting deformation is homogeneous and the tensor gradient of deformation reads

$$
\mathrm{F}=\operatorname{diag}\left(\lambda_{1}, \lambda_{2}\right),
$$

where $\lambda_{1}, \lambda_{2}$ are the deformation in the $X$ and $Y$ direction, respectively.

The transverse balance of forces yields the homogeneous stress

$$
E\left(\lambda_{2}-\frac{1}{\lambda_{2}}\right)=-\kappa \lambda_{1},
$$

where $\kappa$ is the active surface stress generated in the actin cortex. The active surface stress is supposed to depend linearly on the tangential strain.

Equation (6) is an algebraic equation with (admissible) solution

$$
\lambda_{2}=-\frac{\kappa \lambda_{1}}{2 E}+\sqrt{1+\left(\frac{\kappa \lambda_{1}}{2 E}\right)^{2}} .
$$

The longitudinal balance of forces reads:

$$
E\left(\lambda_{1}-\frac{1}{\lambda_{1}}\right)+\alpha \lambda_{1} \lambda_{2}=-\kappa \lambda_{2} .
$$

Using (7), Eq. (8) rewrites as a nonlinear algebraic equation that can be numerically solved by the Newton-Raphson method. 


\section{B. Radially symmetric cell}

The radial symmetry of the problem is exploited introducing the tensor gradient of deformation in polar coordinates

$$
\mathrm{F}=\operatorname{diag}\left(r^{\prime}, r / R\right),
$$

where $r(R)$ is the radial position as a function of the reference coordinate. The balance of forces

$$
\frac{d}{d R} P_{R R}+\frac{1}{R}\left(P_{R R}-P_{\Theta \Theta}\right)=0,
$$

rewrites

$$
\begin{aligned}
& \frac{d}{d R}\left(E\left(r^{\prime}-\frac{1}{r^{\prime}}\right)+\alpha r^{\prime} \frac{r}{R}\right) \\
& +\frac{1}{R}\left(E\left(r^{\prime}-\frac{1}{r^{\prime}}-\frac{r}{R}+\frac{R}{r}\right)+\alpha r^{\prime} \frac{r}{R}\right)=0,
\end{aligned}
$$

to be solved with boundary conditions

$$
r(0)=0,\left.\quad\left(E\left(r^{\prime}-\frac{1}{r^{\prime}}\right)+\alpha r^{\prime} \frac{r}{R}\right)\right|_{R_{0}}=-\left.\kappa \frac{r}{R}\right|_{R_{0}} .
$$

Equation (11) with boundary conditions (12) is a secondorder boundary value nonlinear differential equation. It can be numerically solved by finite differences.

\section{ENERGY DISSIPATION: THE VISCOUS MODEL}

We represent the fluidlike behavior of the cytoskeleton as an infinitely compressible Newtonian fluid undergoing steady flow:

$$
\begin{gathered}
\mathrm{T}=\frac{\mu}{2}\left(\nabla \mathbf{v}+(\nabla \mathbf{v})^{T}\right)+\alpha \mathbf{a} \otimes \mathbf{a}, \\
-\nabla \cdot \mathrm{T}=-\beta \mathbf{v}
\end{gathered}
$$

where $\mathbf{v}(\mathbf{x})$ is the spatial velocity field, $\mathbf{x}$ is the spatial coordinate, $\mathrm{T}$ is the Cauchy stress tensor and $\beta$ is a friction coefficient. In accordance with the literature in the field we assume that the active stress is independent of the strain rate $[7,12,19,20]$. The dissipative force at the right-hand side of Eq. (14) should be understood as modeling the friction among cytosol and cytoskeleton, the fluid and solid phase contained in the lamellipodium. From a structural point of view, the cell is likely a porous medium, the cytosol flowing through the pores of the actin network, which continuously remodels because of polymerization and depolymerization with a strain modulated by the active stress generated by the myosin motors [21].

\section{A. Polarized cell}

For a polarized cell we assume that the velocity is aligned with the direction of the active stress (see Fig. 1), and the force balance equation reads

$$
\begin{aligned}
& T_{x x}=\mu \frac{d v}{d x}+\alpha, \\
& -\frac{d}{d x} T_{x x}=-\beta v,
\end{aligned}
$$

with boundary conditions

$$
v(0)=0, \quad T_{x x}(L / 2)=0 .
$$
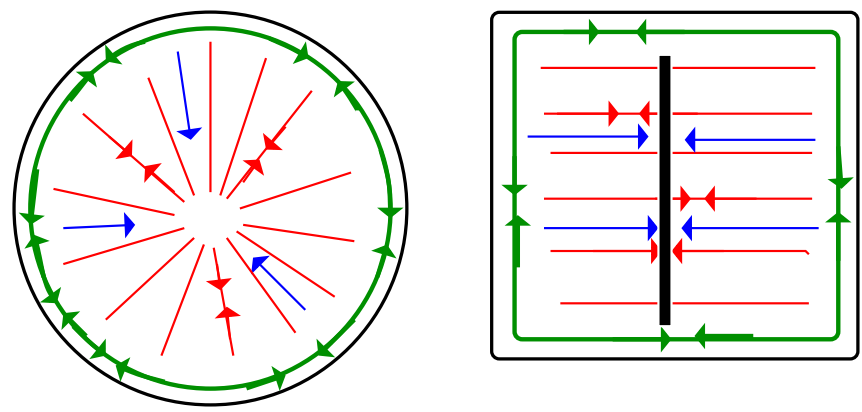

FIG. 1. Drawing of the geometrical configuration of a symmetric (left) and a polarized cell (right). The actin cortex (green) is aligned with the membrane, while the result of the active stress in the cytoskeleton is a tensional pattern (red) radially oriented or along parallel lines. The light blue arrows indicate the direction of the actin flow.

We notice that, in general, the length of the cell (the location of the free boundary) and the velocity of the cytoskeleton are not independent: they must satisfy a compatibility condition that stems after integration of the mass balance equation [19]

$$
\frac{d}{d x}(\rho v)=-\Gamma \rho
$$

yields

$$
\left.\rho_{0} v\right|_{\frac{L}{2}}=-\Gamma \frac{L}{2} \bar{\rho}
$$

where $\rho_{0}$ is the polymerization density at the edge and $\frac{L}{2} \bar{\rho}$ is the total mass. Notably, the relation is independent of the density pattern. In this framework the condition (19) is immaterial because we have the freedom to fix to the total mass so that $L=20 \mu \mathrm{m}$.

Integration of the momentum Eq. (14) with boundary conditions (17) yields

$$
v(x)=-\frac{\alpha}{\sqrt{\beta \mu}} \frac{\sinh \left(\sqrt{\frac{\beta}{\mu}} x\right)}{\cosh \left(\sqrt{\frac{\beta}{\mu}} \frac{L}{2}\right)} .
$$

\section{B. Radially symmetric cell}

For a symmetric cell it is convenient to introduce polar coordinates and the Cauchy stress tensor takes the diagonal form

$$
\begin{gathered}
T_{r r}=\mu \frac{d v}{d r}+\alpha, \\
T_{\theta \theta}=\mu \frac{v}{r} .
\end{gathered}
$$

Balance of forces reads

$$
-\frac{d}{d r} T_{r r}-\frac{1}{r}\left(T_{r r}-T_{\theta \theta}\right)=-\beta v,
$$

with boundary conditions

$$
v(0)=0, \quad T_{r r}\left(r_{0}\right)=0 .
$$


Analogously to the symmetric case, integration of the mass balance equation

$$
\frac{1}{r} \frac{d}{d r}(\rho r v)=-\Gamma \rho
$$

yields the compatibility condition

$$
\rho_{0} v\left(r_{0}\right)=-\Gamma \pi r_{0} \bar{\rho} .
$$

The force balance Eq. (23), using the components of the Cauchy stress (21) and (22) rewrites

$$
\left(\mu v^{\prime}+\alpha\right)^{\prime}+\frac{1}{r}\left(\mu v^{\prime}+\alpha-\mu \frac{v}{r}\right)=\beta v .
$$

Equation (27) with boundary conditions (24) is a second-order boundary value linear differential equation. It can be numerically solved by finite differences and fixed point iterations.

\section{ENERGY STORAGE AND ENERGY DISSIPATION}

The solution of the equation of balance of forces illustrated in the sections above allow a quantitative evaluation of the total energy stored and dissipated in the system, depending on the geometrical configuration. At equilibrium the total mechanical energy stored by the material is equal to the work of (surface and bulk) active forces. In a polarized cell it is

$$
W_{p}=L_{o}^{2} \frac{E}{2}\left[\lambda_{1}^{2}+\lambda_{2}^{2}-2 \log \left(\lambda_{1} \lambda_{2}\right)-2\right],
$$

whereas for a symmetric cell it is

$$
W_{s}=2 \pi \int_{0}^{R_{0}} \frac{E}{2}\left(\left(r^{\prime}\right)^{2}+\left(\frac{r}{R}\right)^{2}-2 \log \left(r^{\prime} \frac{r}{R}\right)-2\right) R d R .
$$

The total mechanical energy dissipated by the material is $\int T \cdot D$, where $D$ is the strain rate tensor; the total energy dissipated by the system includes the power spent by the friction force plus (minus) the power of the internal active forces. After multiplication of the momentum equation times $\mathbf{v}$ and integration by parts [22] for a polarized cell one gets

$$
\dot{K}_{p}=2 L \int_{0}^{L / 2}\left(\mu\left(\frac{d v}{d x}\right)^{2}+\beta v^{2}+\alpha \frac{d v}{d x}\right) d x,
$$

analogously, for a symmetric cell,

$$
\dot{K}_{s}=2 \pi \int_{0}^{r_{0}}\left\{\mu\left[\left(\frac{d v}{d r}\right)^{2}+\left(\frac{v}{r}\right)^{2}\right]+\beta v^{2}+\alpha \frac{d v}{d r}\right\} r d r .
$$

In Eqs. (30) and (31) it should be noticed that the power of active stress, the last term at the right-hand side, is a source of kinetic energy when the derivative of the velocity versus the outward directed coordinate is negative.

\section{NUMERICAL RESULTS}

Numerical integrations have been performed using the set of physical parameters taken from the literature and listed in Table I. With an abuse of notation, we denote by the same symbols the corresponding two-dimensional (2D) parameters,
TABLE I. Typical values of the physical parameters as taken from the literature.

\begin{tabular}{llll}
\hline \hline Parameter & \multicolumn{1}{c}{ Physical Meaning } & \multicolumn{1}{c}{ Value } & Source \\
\hline$L_{0}, L$ & cell length & $20 \mu \mathrm{m}$ \\
$\alpha$ & cytoskeleton active stress & $1 \times 10^{3} \mathrm{pN} / \mu \mathrm{m}^{2}$ & {$[20]$} \\
$\beta$ & friction coefficient & $1 \times 10^{4} \mathrm{pN} \mathrm{s} / \mu \mathrm{m}^{2}$ & {$[20]$} \\
$\mu$ & viscosity coefficient & $5 \times 10^{5} \mathrm{pN} \mathrm{s} / \mu \mathrm{m}^{2}$ & {$[12]$} \\
$E$ & bulk elastic modulus & $1 \times 10^{4} \mathrm{pN} / \mu \mathrm{m}^{2}$ & {$[23]$} \\
$\kappa$ & cortex active stress & $4 \times 10^{4} \mathrm{pN} / \mu \mathrm{m}$ & {$[24]$} \\
\hline \hline
\end{tabular}

obtained after integration of the 3D equations along the cell thickness $(1 \mu \mathrm{m})$.

In Fig. 2 are plotted the stored energy, as calculated by numerical integration of Eqs. (28) and (29), for a squared (black) and circular (gray) cell versus the active stress $\alpha$. The plot exhibits an energy crossover around $1 \times 10^{3} \mathrm{pN} / \mu \mathrm{m}^{2}$ : the minimum energy configuration is the polarized configuration for small $\alpha$, while the squared configuration has smaller elastic storage for large $\alpha$. A possible interpretation of such a behavior is that for small $\alpha$ the active stress in bulk plays a secondary role versus the surface cortex tension: as a polarized cell has a longer boundary, its corresponding energetic contribution dominates. Conversely, for large activity the molecular motors in a symmetric cell can strain the cytoskeleton without the geometrical constraints intrinsic to the rounded geometry and the curve is essentially traced by the corresponding elastic energy.

Figure 3 shows the integral of dissipated kinetic energy in the system for a for a squared (black) and circular (gray) cell versus the active stress $\alpha$. The polarized configuration is always more dissipative for any $\alpha$.

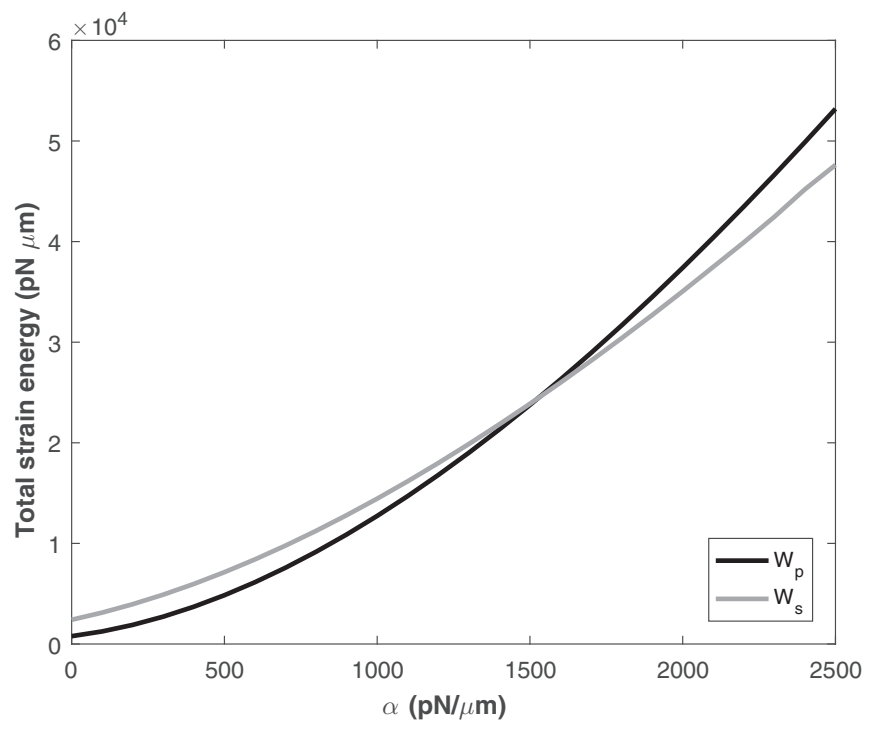

FIG. 2. Plot of the total energy stored by the system vs the active stress $\alpha$ : polarized (black) and nonpolarized (gray) configuration. 


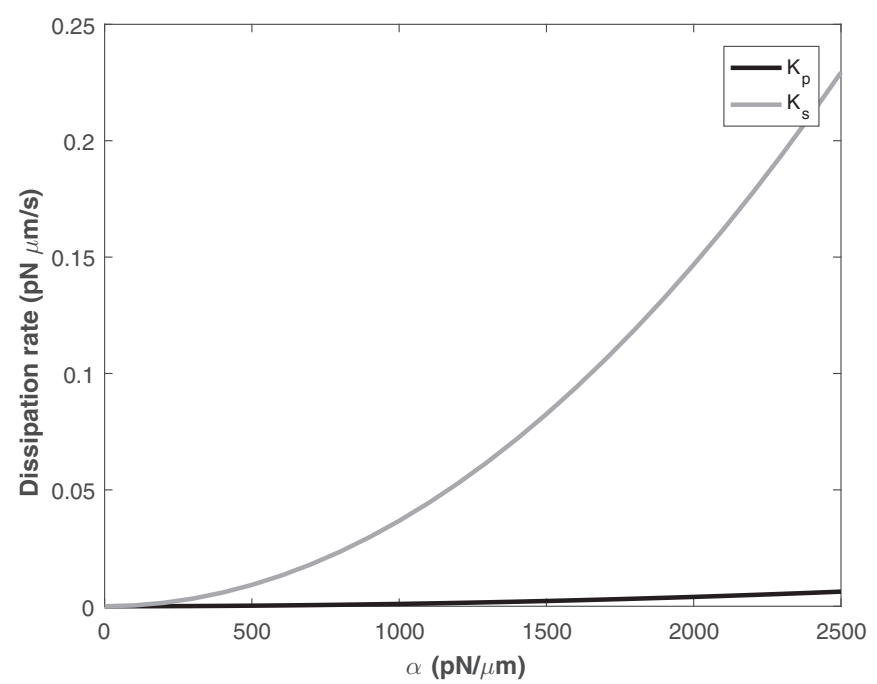

FIG. 3. Plot of the total power spent by the internal and external forces vs the active stress $\alpha$ : polarized (black) and nonpolarized (gray) configurations.

\section{DISCUSSION OF THE RESULTS AND FINAL REMARKS}

In this paper we have addressed a quantitative comparison between the energy stored and dissipated per unit time by a living cell at rest, in its symmetric and polarized configurations. The elastic energy is supposed to be due to the strain of the actomyosin in bulk, while passive contribution due to the strain of the actin cortex at the surface of the cell is neglected. Conversely, both active stress in the cytoskeleton (bulk) and in the cortex (surface) are taken into account.

Bulk viscosity and cytosol-skeleton friction are responsible for the energy dissipation. The rationale behind this decoupled analysis is that at the steady state a nonlinear elastic model can account for the energy stored in the material, while a viscous fluid model captures the dissipation. Our physical intuition is the physical representation of the cytoskeleton as a Kelvin-type material lumped element: a spring and a dashpot in series. Whatever (nonlinear) are the physical laws of the lumped elements, at the long-time equilibrium the two contributions can be separately evaluated. It is worth to remark that a purely fluid model with a pressurelike energetic storage would not be sufficient to capture the full physics of the cytoskeleton; even in an elastic solid with circular symmetry the stress tensor is not spherical, radial and hoop stress can be different.

Biophysical implications of the results illustrated in Sec. V are to be discussed versus the experimental observations. Cells with different geometrical configurations and about the same area result in a similar amount of mechanical (elastic) work performed on the environment [25]. In the same vein, numerous experimental groups have found that the strain energy is proportional to the spread area for a wide range of cell types [26]. These observations suggest that cells usually span an activation range not far from the critical one, corresponding to the intersection between the two curves plotted in Fig. 2.
The energetic gap that occurs for small $\alpha$ is due, in the present theory, to geometric reasons only: a longer perimeter generates a stronger contribution from the cortex. There is, however, another physical effect that could amplify such a difference: if the contraction of the myosin motors across actin fibers favors parallel versus nonparallel configuration, an extra term proportional to $\left|\nabla \mathbf{a}_{0}\right|^{2}$ should be accounted for. Such a contribution, reminiscent of the distortion free energy of liquid crystals, in our setting would be constant for a symmetric cell, while vanishing for a polarized cell. In the absence of a clear evidence of an energetic cost for fibers twist, we do not include such a contribution in the model; we just notice that it would further support and amplify our findings, possibly displacing the critical value to the right in the $\alpha$ axis.

The crossover reported in Fig. 2 tells us that polarization gives the minimum energy configuration for low activity of the motors (small $\alpha$ ), while stronger activity makes symmetry energetically convenient. Intriguingly, this result is in accordance with experiments, where the polarization switch is interpreted as a competition between active stress and dendritic growth [4].

In the same vein, Fig. 3 tells us that the symmetric system is always more consuming (in terms of mechanical energy) than the polarized one. A possible interpretation of these results can be framed in a control perspective: a cell is a mechanical system that places itself in a most dissipative configuration, which is only locally of minimum energy, because it allows to reach more easily other more stable configurations among the many possible ones. In a biophysical perspective, a rounded cell at rest, with a randomly organized cytoskeleton, stores and spends more energy, but this cost is rewarded by a cheaper and faster accessibility to polarization according to a direction dictated by external signals, possibly of chemotactic type. In other words, our results suggest that a symmetric cell is an energetically expensive metastable state that allows the access to infinite lower-energy stable configurations, at variance of the direction of polarization.

The interpretation above is reminiscent of the maximum dissipation principle [27], here recast as in a geometrical setting. In this perspective, keeping the living cell with an activity level around the critical $\alpha$ might purposely allow steering the system from the metastable to a stable state.

The present work compares well with Kozlov and Mogilner [15] who exploit energy competition arguments in cell polarization as we do. However, while they study the energy landscape as a function of shape only, we investigate the mutual balance between strain energy storage and dissipation depending on the active stress magnitude. Our approach, based on a numerical integration of two-dimensional force balance equations enforced by symmetry arguments can therefore be considered as complementary to their work.

The maximum dissipation principle could also be exploited to devise the dynamics from one stable configuration to another one [28]. The mechanism of transition among metastable and stable states requires nonlinearity; this is likely due to the positive feedback between the transport of molecular motors and the active stress, produced by the molecules themselves [29]. This is a further step to be investigated in two dimensions. 
[1] M. Murrell, P. W. Oakes, M. Lenz, and M. L. Gardel, Nature Rev. Mol. Cell Biol. 16, 486 (2015).

[2] A. B. Verkhovsky, T. M. Svitkina, and G. G. Borisy, Curr. Biol. 9, 11 (1999).

[3] K. Carvalho, J. Lemière, F. Faqir, J. Manzi, L. Blanchoin, J. Plastino, T. Betz, and C. Sykes, Phil. Trans. R. Soc. B 368, 20130005 (2013).

[4] A. J. Lomakin, K.-C. Lee, S. J. Han, D. A. Bui, M. Davidson, A. Mogilner, and G. Danuser, Nature Cell Biol. 17, 1435 (2015).

[5] J. Stricker, T. Falzone, and M. L. Gardel, J. Biomech. 43, 9 (2010).

[6] B. Rubinstein, M. F. Fournier, K. Jacobson, A. B. Verkhovsky, and A. Mogilner, Biophys. J. 97, 1853 (2009).

[7] F. Jülicher, K. Kruse, J. Prost, and J.-F. Joanny, Phys. Rep. 449, 3 (2007).

[8] P. A. Pullarkat, P. A. Fernández, and A. Ott, Phys. Rep. 449, 29 (2007).

[9] L. Wolff, P. Fernández, and K. Kroy, PloS ONE 7, e40063 (2012).

[10] D. Ambrosi and P. Ciarletta, Int. J. Nonlinear Mech. 56, 56 (2013).

[11] L. Giomi and A. DeSimone, Phys. Rev. Lett. 112, 147802 (2014).

[12] K. Kruse, J.-F. Joanny, F. Jülicher, and J. Prost, Phys. Biol. 3, 130 (2006).

[13] E. Tjhung, D. Marenduzzo, and M. E. Cates, Proc. Natl. Acad. Sci. 109, 12381 (2012).

[14] A. E. Carlsson, Phys. Rev. E 74, 051912 (2006).
[15] M. M. Kozlov and A. Mogilner, Biophys. J. 93, 3811 (2007).

[16] N. Smith, D. Nickerson, E. Crampin, and P. Hunter, Acta Numerica 13, 371 (2004).

[17] M. Lenz, Phys. Rev. X 4, 041002 (2014).

[18] M. F. Fournier, R. Sauser, D. Ambrosi, J.-J. Meister, and A. B. Verkhovsky, J. Cell Biol. 188, 287 (2010).

[19] D. Ambrosi and A. Zanzottera, Physica D 330, 58 (2016).

[20] K. Larripa and A. Mogilner, Physica A 372, 113 (2006).

[21] E. Moeendarbary, L. Valon, M. Fritzsche, A. R. Harris, D. A. Moulding, A. J. Thrasher, E. Stride, L. Mahadevan, and G. T. Charras, Nature Mater. 12, 253 (2013).

[22] L. D. Landau and E. M. Lifshitz, Fluid Mechanics (Pergamon Press, London, 1959).

[23] L. Lu, S. J. Oswald, H. Ngu, and F. C.-P. Yin, Biophys. J. 95, 6060 (2008).

[24] A. Diz-Muñoz, D. A. Fletcher, and O. D. Weiner, Trends Cell Biol. 23, 47 (2013).

[25] P. Guthardt Torres, I. B. Bischofs, and U. S. Schwarz, Phys. Rev. E 85, 011913 (2012).

[26] N. W. Goehring, P. K. Trong, J. S. Bois, D. Chowdhury, E. M. Nicola, A. A. Hyman, and S. W. Grill, Science 334, 1137 (2011).

[27] P. Fratzl, F. D. Fischer, and J. Svoboda, Phys. Rev. Lett. 95, 195702 (2005)

[28] R. C. Dewar, J. Phys. A 38, L371 (2005).

[29] P. Recho, T. Putelat, and L. Truskinovsky, J. Mech. Phys. Solids 84, 469 (2015). 